\title{
TRANSVERSALIDAD E IMPACTO DE GÉNERO: DE LAS POLÍTICAS A LOS PROGRAMAS. ESTUDIO DE CASO EN LAS ISLAS CANARIAS
}

\section{Gender mainstreaming and impact: from policies to programs. A case study in the Canary Islands}

\author{
Carmen Ascanio Sánchez \\ cascanio@ull.edu.es \\ Universidad de La Laguna - España
}

Recibido: 01-10-2016

Aceptado: 04-05-2017

\section{Resumen}

La transversalidad de género es un concepto ampliamente difundido y defendido que, sin embargo, también ha sido criticado por su ambigüedad y polisemia. Si bien este enfoque debe atravesar los diferentes niveles -desde las políticas a los programas-, la práctica muestra un modelo dual y desarticulado entre, por una parte, los marcos estratégicos y por otro, la puesta en marcha de acciones específicas. A partir de un estudio de caso en las Islas Canarias y de una evaluación de impacto de género (Acogida temprana), se identifican debilidades, oportunidades y contradicciones en la práctica de la transversalidad, mostrando como a través de este tipo de evaluaciones pueden desarrollarse estrategias y herramientas para implementar la articulación entre los niveles.

Palabras clave: Transversalidad, impacto de género, acogida temprana, igualdad de género.

\begin{abstract}
"Gender mainstreaming" is an oft-used, broadly supported term that, however, has also been criticized for being an ambiguous word with multiple meanings. In theory, mainstreaming should cut across all different levels -from policies to programs-, but practice has shown that the model tends to be both dual and disjointed, focusing on strategic frameworks on the one hand and the implementation of specific actions on the other. Using a case study on the Canary Islands and a gender impact assessment (in early childcare), this paper identifies weaknesses, opportunities and contradictions in mainstreaming as it plays out in practice, showing how this type of assessment can be used to develop strategies and tools for linking up the various levels of implementation.
\end{abstract}

Keywords: Mainstreaming, gender impact, early childcare, gender equality. 


\section{Introducción}

La búsqueda de la equidad en diversos ámbitos de lo social, ha sido uno de los focos de atención de las políticas de organismos internacionales y de la Unión Europea en los últimos decenios; uno de sus vértices más relevantes es el de la eliminación de las desigualdades y discriminaciones entre mujeres y hombres. A partir de los años setenta del siglo XX, ha habido un importante consenso político respecto a la legitimidad de las demandas de las mujeres, aunque también se han producido controversias sobre los modelos a seguir y el papel de los organismos públicos. De hecho, se han desarrollado diversas estrategias, de las que destacan las políticas de igualdad de trato, las acciones positivas y el denominado mainstreaming o transversalidad (Consejo de Europa, 1999).

Este último concepto ha sido ampliamente difundido y defendido, pero, también, criticado por su ambigüedad y polisemia. Del mismo modo, se ha visto atravesado por los debates de la interseccionalidad, que opera con diversos tipos de desigualdad (Bustelo, 2009). Lo cierto es que el enfoque de la transversalidad implica deslizarse entre los distintos niveles, desde las políticas a los programas, aunque la práctica muestra un modelo dual: por una parte, los marcos estratégicos y, por otra, la puesta en marcha de programas y acciones específicas. Este artículo defiende, primero, la necesidad de articular estos niveles y dualidades en las políticas de igualdad de género, implementando el mainstreaming en las acciones específicas a través del ciclo de identificación, formulación de políticas, implantación y evaluación. Segundo, que desde el análisis empírico se puede afinar y describir esos múltiples significados de la transversalidad. Esto puede convertirla en una potente estrategia desde la que identificar debilidades, oportunidades y contradicciones en su práctica y, por tanto, para implementar la articulación entre los niveles. Lo anterior se mostrará a partir de un proyecto que se inició con la evaluación de varios programas educativos de la Consejería de Educación, Cultura, Deportes y Universidades del Gobierno de Canarias ${ }^{1}$, y ha continuado con el seguimiento de algunas acciones, en especial el de Acogida temprana. El foco de esta investigación ha sido la evaluación del impacto de género, es decir en los efectos de determinadas políticas en la vida de mujeres y hombres.

El texto consta de tres partes: en la primera, se plantea el concepto de transversalidad con el foco de atención puesto en los avances del enfoque feminista y la economía comprensiva y, por tanto, en la operatividad de metodologías multiestratégicas. El siguiente bloque, explica el estudio de caso y analiza con brevedad el contexto de análisis, el proyecto realizado y la acción

\footnotetext{
${ }^{1}$ Consejería de Educación, Cultura, Deportes y Universidades en los inicios del proyecto; Consejería de Educación y Universidades en la actualidad. En adelante "Consejería de Educación". Proyecto: "Análisis y evaluación de impacto de género en políticas públicas educativas (IGECAN)", Consejería de EducaciónULL, 2008-2009. El equipo inicial estaba formado por: Carmen Ascanio (directora) y la coordinadora y becarias: Nuria Roldan, Leticia Fajardo y Yolanda Márquez. El análisis y seguimiento se ha realizado por parte de la autora.
} 
seleccionada. Por último, se plantea la utilidad de estas evaluaciones para identificar la práctica de la transversalidad, centrándonos en aspectos clave que deben servir de referencia.

\section{Transversalidad, metodologías y herramientas}

Las estrategias a seguir para la eliminación de las desigualdades entre mujeres y hombres, así como el papel de los organismos públicos han sido asuntos controvertidos. Un primer consenso ha sido la institucionalización del feminismo, mayoritariamente a través de organismos de igualdad (Stetson y Mazur, 1995). Éstos se comienzan a crear en España a partir de los años ochenta del siglo XX, siendo los encargados de desarrollar diversos tipos de políticas, como las de igualdad de trato o las acciones positivas. Ya en los años noventa, a partir de la Plataforma de Acción de la IV Conferencia Mundial sobre Mujeres de Naciones Unidas (1995), surge la estrategia del mainstreaming, que ha conseguido otro importante consenso. En un informe de 1998, el Consejo de Europa la definía como: "El mainstreaming de género es la organización (reorganización), el avance, el desarrollo y la evaluación de los procesos políticos, de modo que una perspectiva de igualdad de género, se incorpore en todas las políticas, a todos los niveles y en todas las etapas, por los actores normalmente involucrados en la adopción de medidas políticas" (Consejo de Europa, 1999: 26).

Esta definición implica a todos los niveles y etapas del ciclo de las políticas públicas, así como la reorganización de la práctica política en cada ámbito y/o institución. Como señala Lombardo (2003), esto requiere importantes cambios: en el mismo concepto de igualdad existente, en la incorporación de esta perspectiva a la agenda política dominante, en la participación de las mujeres en instituciones y procesos de tomas de decisiones, en las prioridades (por ejemplo, las políticas sociales y familiares) y, por último, en la necesidad de cambios en las culturas institucional y organizativa. Ciertamente, en los dos últimos decenios se ha avanzado en estas necesarias transformaciones, aunque también se perciben procesos de paralización e, incluso, de involución. Por dicho motivo, defendemos que cualquier proceso con enfoque de transversalidad de género debe partir del marco teórico emprendido por el feminismo y por la economía comprensiva, así como los aportes recientes sobre el bienestar humano (Bognar, 2005; Nussbaum, 2012; Sen, 2007); lo contrario significaría quedarnos en meras retóricas sobre la transversalidad que solo sirven para cubrir el expediente.

De hecho, el bagaje -teórico, metodológico y empírico- con el que actualmente se cuenta, está claramente influenciado por la entrada de mujeres en el ámbito del conocimiento académico, así como por la densidad y continuidad de su trabajo. La mayor parte de esta producción se ha centrado en temas como la familia, la producción/reproducción, el cuidado infantil y la incorporación de la mujer al mercado de trabajo. Estos análisis muestran como los anteriores 
modelos no daban cuenta de las estructuras de dependencia, tradición y poder, desde las que se han estructurado las relaciones de género (Ferber y Nelson, 2004).

Efectivamente, las sociedades funcionan a partir de procesos de "producción" y "reproducción", sea de bienes y personas como de relaciones (Greenstein, 2000). Por tanto, avanzar en la conciliación de la vida laboral y familiar implica una definición del trabajo diferente: como toda actividad básica para la reproducción propia o de la sociedad. Esto hace que sea fundamental tener en cuenta los tipos de trabajo (el remunerado/no remunerado y formal/informal) y, dependiendo de los asuntos abordados, su interrelación con los sectores básicos, de visibilidad histórica diferencial: el sector público, el privado, el doméstico y el que se denomina sector de ONG por parte de UNIFEM (2000). Estos cuatro sectores están conectados por canales, tanto del mercado como de la producción/reproducción de las sociedades en general. Valores de tipo comercial fluyen desde el sector privado, de tipo regulador del sector público (normas, leyes, etc.); otros relacionados con la subsistencia proceden del sector doméstico que se ocupa de la satisfacción de las necesidades básicas. Por último, valores de reciprocidad y cooperación vienen del sector de las ONGs/Asociativo. Las dinámicas de todos estos sectores se insertan en las formas jerárquicas y excluyentes de la globalización de las desigualdades. Esto se visibiliza en la dual división del trabajo: por una parte, que el trabajo de cuidado no remunerado, voluntario e informal remunerado y no remunerado tienda a ser realizados mayoritariamente por mujeres; por otra, que el trabajo formal remunerado en los sectores privado, público y de ONG sea realizado mayoritariamente por hombres. En la esfera laboral y doméstica esta realidad social ha tenido importantes consecuencias. Por ejemplo: que el acceso y promoción al mercado de trabajo haya estado supeditado a las responsabilidades familiares de las mujeres (Booth et al. , 2002), especialmente bajo el rol de "madre" (Artis y Pavalko, 2003; Hugher, 2006), o que el mismo mercado de trabajo -y parte de la sociedad- demande mayor disponibilidad por parte de los varones, restando atención al ámbito doméstico (Noonan, 2001), o la gestión diferencial del tiempo privado y profesional (Aliaga, 2006; Mclanahan y Percheski, 2008; Torns, Borrás, Moreno y Recio, 2008). En Europa ha habido una importante producción académica sobre los sistemas de cuidado infantil, donde se plantean diversos modelos ubicados en el recorrido del dualismo clásico: el del hombre como principal aporte económico de la familia y el de la mujer como ama de casa (Gornick y Meyers,2003; Haddad, 2002; Krapf, 2014; OECD, 2005; Vandenbroeck, Geens y Berter, 2014).

Por ello, una revisión con perspectiva de género requiere de un análisis complejo (Htun y Weldon, 2010) que interrelacione aspectos estructurales (como los modelos de Estado y bienestar y los regímenes de género), con el desarrollo de los organismos y agentes de igualdad en cada contexto (movimiento feminista, evolución de los diferentes actores, etc.). De hecho, la comparativa entre países muestra la relevancia de tradiciones y trayectorias nacionales y/o territoriales, a pesar de situaciones económicas similares: diferentes percepciones y prácticas 
sobre el cuidado infantil, el trabajo de las mujeres y la educación, producen divergencias en las políticas del cuidado y la gestión del tiempo (Folbre, 2009; Sayer, 2005).

En síntesis, a pesar de los discursos y las políticas de igualdad, se demuestra que la estructura de los mercados, la misma definición de lo que es trabajo, el tiempo y los roles siguen estableciendo una brecha entre mujeres y hombres que explica las múltiples paradojas del feminismo (Allen, 2005). En este sentido, la re-definición de conceptos como "desarrollo humano" o la ampliación de otros como el de "eficacia" resultan clave para comprender las conexiones entre las políticas públicas de educación, el cuidado infantil y el trabajo de las mujeres (Ainscow, Dyson, Goldrick y West, 2013), especialmente en países con modelos polarizados (Goñi-Legaz, Ollo-Lopez y Bayo-Moriones, 2010).

Para llevar a cabo toda esta serie de transformaciones se requiere de estrategias específicas en cada ámbito, así como de evaluaciones que interrelacionen políticas y programas, sus debilidades, oportunidades y contradicciones desde el enfoque de la transversalidad. Diversas herramientas tienen ya un importante recorrido en los últimos decenios: informes/evaluaciones de impacto, indicadores y presupuestos de género, etc. Por ejemplo, los primeros han sido desarrollados en especial por los organismos internacionales, como sustento teórico y político de los denominados planes de igualdad.

Los presupuestos de género también han sido clave para el diseño de políticas públicas con enfoque transversal: ya no se los considera como simples agregados financieros, neutrales al género, sino -al contrario- instrumentos que afectan de modo desigual a la vida de mujeres y hombres (Cagatay, Keklik, Lal y Lang, 2000). En el último decenio, el análisis presupuestario ha sido objeto de atención por parte de organismos internacionales, nacionales y locales, aunque desde los años ochenta del siglo XX se venían desarrollando numerosas experiencias: en Australia, Reino Unido, Canadá, Sudáfrica y otros países de América Latina, África y el ámbito de la Commonwealth (Budlender y Hewitt, 2003).

En Europa también se han realizado estudios, con enfoques y articulación diversa ente lo local y lo nacional, que han tenido en común la participación de grupos y asociaciones de mujeres. En España estas iniciativas han sido desarrolladas, en especial, en el País Vasco (Emakunde, Instituto Vasco de la Mujer) y en Andalucía (Consejería de Hacienda de la Comunidad Autónoma), dónde se ha realizado una destacable labor estadística en materia de desagregación por sexo. En los últimos años han aumentado este tipo de experiencias locales y autonómicas (Biencinto y González, 2010), aunque son escasos los estudios empíricos, participativos y comparativos.

Lo cierto es que el interés internacional que ha suscitado este enfoque ha provocado su rápida evolución y cambios de conceptos, ámbitos de análisis y diseño de herramientas (Budlender, Sharp y Allen, 1998; Elson, 2002). Algo similar ocurre con los indicadores sensibles al género que también tienen una densa trayectoria, en especial a partir de las recomendaciones de la IV conferencia mundial sobre la mujer (CEPAL, 2006). 


\section{De las políticas a los programas}

\subsection{Las políticas de igualdad}

En España, a partir de la etapa democrática, ha habido un continuo interés desde las administraciones por desarrollar políticas de igualdad, aunque el énfasis y compromiso ha variado dependiendo de los gobiernos centrales y autonómicos. De hecho, la gestión de políticas de igualdad se ha planteado de forma descentralizada y horizontal, en los tres niveles de la Administración (central, autonómico y local). En todo caso, las comunidades autónomas han tenido un importante papel, desarrollando organismos específicos y planes de igualdad (Alonso, 2015); su comparativa muestra diferencias relevantes: ser organismo autónomo o no, su ubicación en el organigrama institucional, sus planes y enfoques, etc. Con toda esta importante trayectoria en materia de igualdad, la crisis actual ha significado una evidente ruptura de compromisos anteriores, tal y como muestran las últimas reformas gubernamentales y el análisis de los primeros datos (Gálvez y Torres, 2010): las políticas de conciliación ya no parecen ser una prioridad. En la Comunidad Autónoma Canaria ha habido una trayectoria de fuerte compromiso con políticas de igualdad de oportunidades, incluyendo este objetivo en su Estatuto de Autonomía (1982). En 1994 se crea el Instituto Canario de la mujer que en el 2010 pasa a denominarse Instituto Canario de Igualdad (ICI), adscrito a la Consejería de Presidencia, Justicia e Igualdad del Gobierno de Canarias. El organismo ha desarrollado hasta la actualidad tres planes de igualdad entre mujeres y hombres (1995-1996, 1997-2000 y 2003-2006) y la actual Estrategia para la Igualdad de Mujeres y Hombres 2013-2020. A partir II Plan se incluye el mainstreaming como mandato. El III Plan (Gobierno de Canarias, n.d.) cuenta con un importante diagnóstico de la realidad canaria, sistematiza herramientas de trabajo y menciona la necesidad de introducir la perspectiva de género en todas las políticas y planes. Dicha estrategia es un denso documento que abarca numerosas áreas de la realidad canaria, con nueve ejes estratégicos, donde se plantea la integración de la perspectiva de género en todas las políticas generales y sectoriales, así como del desarrollo de herramientas de evaluación del impacto de género

\subsection{El proyecto IGECAN}

El proyecto IGECAN (2008-2009) surge del interés de la Consejería de Educación, de desarrollar una línea de trabajo sobre análisis y evaluación de impacto de género, plasmándose en un proyecto-convenio entre el organismo y la Universidad de La Laguna. El contacto inicial se estableció a través de altos cargos (mujeres) con elevada sensibilidad en asuntos de género. Desde el comienzo se planteó como un proyecto externo, sin participación del personal del organismo implicado, aunque con la máxima colaboración en el aporte de datos. Hubo intentos de implicar a diversos agentes internos, pero no dieron fruto más allá de colaboraciones puntuales. 
En la primera etapa, el proyecto se planteó cuestiones básicas del conocimiento de la realidad canaria. En la segunda etapa, el centro de atención se trasladó al ámbito microsocial; por dicho motivo, se puso e foco de atención en las interrelaciones entre lo macro y lo micro, así como en cuestiones clave presente en todos los programas: los sistemas de cuidado infantil, los tipos de trabajo de mujeres y hombres, y la construcción de estereotipos. La metodología utilizada ha tenido un enfoque multiestratégico, con fuerte aporte cualitativo y de trabajo de campo. La misma ha estado presente a lo largo de todo el proceso: desde el marco general de los presupuestos públicos, al análisis interno de los programas y la construcción de indicadores de género. Los ocho programas seleccionados fueron: el de acogida temprana, el de transporte y comedor, el de actividades extraescolares, el de refuerzo escolar, el de tarjeta escolar (adquisición de libros escolares), el de formación profesional, el de deportes y el de cultura denominado "septenio". Para su clasificación inicial se utilizó una tipología ${ }^{2}$ (Villota, 2008) que destacó como único programa en igualdad de oportunidades (PIO) al de Acogida Temprana ${ }^{3}$.

El marco general para el inicial análisis de los programas ha sido el Presupuesto General de la Comunidad Autónoma de Canarias, comparando las diferentes áreas de educación/cultura, los programas y las acciones más relevantes para la igualdad de oportunidades. La evaluación de cada programa ha incluido un análisis interno y diacrónico (seguimiento y cambios), la detección de los ámbitos prioritarios para la elaboración de indicadores de género y el análisis de fuentes y datos sobre el mismo (estadísticas, normas y datos cualitativos). Las fuentes y técnicas utilizadas han dependido de las fases: en la primera se realizó un importante esfuerzo por recopilar datos y estudios que ofrecieran un marco comprensivo sobre la realidad educativa, el cuidado infantil y el mercado laboral entre mujeres y hombres. A partir de aquí se inició el diseño de la fase cualitativa donde se realizaron, básicamente, etnografías selectivas y entrevistas/grupos de discusión a agentes clave de cada uno de los programas; todo ello en la isla de Tenerife, dónde radicaba el equipo de investigación. Las etnografías selectivas se realizaron en centros educativos, en las mismas reuniones con diversos agentes, en el seguimiento de actividades, etc., con el foco de atención puesto en el cuidado infantil (tareas, desayunos, comedores,), tipos/tiempos de trabajo y la detección de estereotipos. Las entrevistas y grupos de discusión han tenido como objetivo el identificar tanto las brechas de género como los problemas y debates en cada uno de los programas ${ }^{4}$. Finalizado el

\footnotetext{
${ }^{2}$ Se distingue entre PIO (programas de igualdad de oportunidades), PM (programas mixtos o aquellos que tienen alguna actividad relacionada con la igualdad de oportunidades), PI (programas implícitos, o sea no específicamente orientados a la igualdad de oportunidades pero que provocan un claro impacto diferenciado entre mujeres y hombres) y PA (programas administrativos no orientados).

${ }^{3}$ Entre los programas destacaban los de tipo implícito (PI): el programa de Refuerzo escolar, el de Formación profesional, el de Deportes y el de cultura "Septenio". Dos son PM: el de Transporte y el de Comedor, y el de Actividades extraescolares; sin embargo, éste surgió como PI y con la modificación de su normativa (2007 y 2009) paso a ser un PM, encaminado a objetivos relacionados con la conciliación de la vida familiar y laboral. El programa de Tarjeta escolar para la adquisición de libros escolares es el único PA, actualmente desaparecido. ${ }^{4}$ En la primera etapa se realizaron grupos de discusión en cada programa y entrevistas (2009) a miembros de las Asociaciones de Madres y Padres de Alumnos (AMPAs), 20 madres y padres miembros de las mismas, a gestores de centros educativos (4), a orientadores escolares (16) y a gestores de las empresas privadas (2).
} 
proyecto-convenio se ha realizado un seguimiento, especialmente para el programa de Acogida temprana, con breves indagaciones de campo (2013-2016).

\section{El programa de acogida temprana}

\subsection{Diagnóstico y resultados}

Se ha seleccionado el ejemplo de este programa por tres razones: es el único de Igualdad de Oportunidades (PIO), se centra en ámbitos claves como el cuidado infantil y el mercado de trabajo y, por último, ha habido un seguimiento hasta la actualidad. El programa de Acogida temprana surge, paradójicamente, en plena crisis. La orden de su creación (Consejería de Educación, 2008) indica que su objetivo es apoyar la conciliación de la vida familiar y laboral a través de la apertura de los centros escolares entre una a dos horas antes del inicio de la jornada lectiva, de modo que los progenitores puedan hacer compatible el horario laboral y el escolar. En esta primera etapa se planteó como un programa con medidas universales, beneficiando a toda la población. La evaluación del programa se solicitó a posteriori, en el segundo año de su recorrido, desarrollándose en dos fases: la inicial, de descripción y análisis de actividades e identificación de los agentes clave (directos e indirectos) y la final, interrelacionado todo lo anterior con los tipos de trabajo/sectores y sus consecuencias en el impacto de género.

A partir del enfoque multiestratégico mencionado se identificó y analizó el impacto en agentes y sectores implicados en el programa (sector público, privado, doméstico y el de las ONGs/Asociaciones). Teniendo en cuenta las características de la medida en las Islas, el impacto directo de la misma es el número de niños y niñas y el de las madres/padres que se benefician del servicio. En el primer año de aplicación (2008) participó en el programa un 11\% del total de alumnado de centros de educación infantil y primaria (CEIP) y de enseñanza secundaria obligatoria (ESO). Respecto a los progenitores, habría que mencionar que en la mayor parte de los contextos dónde esta medida ha sido aplicada, el beneficio de la misma ha sido en especial para las madres (England, 2005); algo lógico, ya que son las mujeres las que tienen evidentes dificultades para conciliar su participación en el mercado de trabajo remunerado con el trabajo de cuidado a terceros. Sin embargo, la Consejería de Educación, apenas contaba con datos del grupo doméstico en las fichas de inscripción del alumnado del programa, a pesar de que la medida estaba especialmente dirigida a progenitores insertados en el mercado laboral formal y remunerado.

También se tuvo en cuenta el impacto indirecto en el resto de agentes: Asociaciones de Madres y Padres de Alumnos (AMPAs) ${ }^{5}$, el profesorado voluntario y las empresas adjudicatarias del servicio. En realidad, en las distintas directrices de la Consejería de Educación, quedaba

\footnotetext{
${ }^{5}$ AMPAs, en adelante.
} 
patente que se pretendía priorizar que esta medida fuera ejecutada por profesorado voluntario. La realidad mostró que la participación del mismo ha sido irrelevante. La responsabilidad de esta acción ha tenido que ser asumida, mayoritariamente, por las AMPAs, que reciben una subvención del gobierno autónomo para contratar empresas y/o monitores. En la primera fase señalada, la responsabilidad de la gestión ha sido mayoritariamente para las AMPAs (74\%), el profesorado voluntario $(21 \%)$ y el resto de agentes (profesorado nombrado y mixto, $5 \%$ ). En cuanto a las empresas adjudicatarias, se carecía de información sobre el perfil del personal, planes de igualdad y otros datos; en el trabajo de campo tampoco se ofrecieron datos más allá de la contabilidad general de sus trabajadores/as.

Los objetivos siguientes se centraron en identificar los tipos de trabajo y los sectores implicados en el desarrollo del programa de Acogida Temprana. Se han visibilizado dos tipos de trabajo: el formal remunerado y el voluntario. Junto a estos, especialmente a partir del trabajo de campo, se ha identificado determinadas estrategias laborales que se pueden denominar como trabajo mixto/hibrido (Rodríguez, 2013) o de trayectoria mixta; en concreto, algunos casos de contrataciones de las empresas adjudicatarias que recaen en miembros (mujeres-madres) del AMPAs de su mismo colegio. En estos casos se nos explicó la dificultad de encontrar personal para contratos de gran precariedad (horarios discontinuos, múltiples tareas no siempre contratadas, bajos salarios, etc.); por tanto, se debe acudir a ámbitos (AMPAs) donde ya se trabajaba gratis, en tareas conocidas, siempre en relación a sus propios hijos o vecinos y en sus mismos espacios de convivencia. La interrelación analizada entre trabajo y sectores, siguiendo a UNIFEM (2000), se puede simplificar del siguiente modo:

\section{Gráfico 1. Interrelación de los sectores económicos en el Programa de Acogida Temprana}

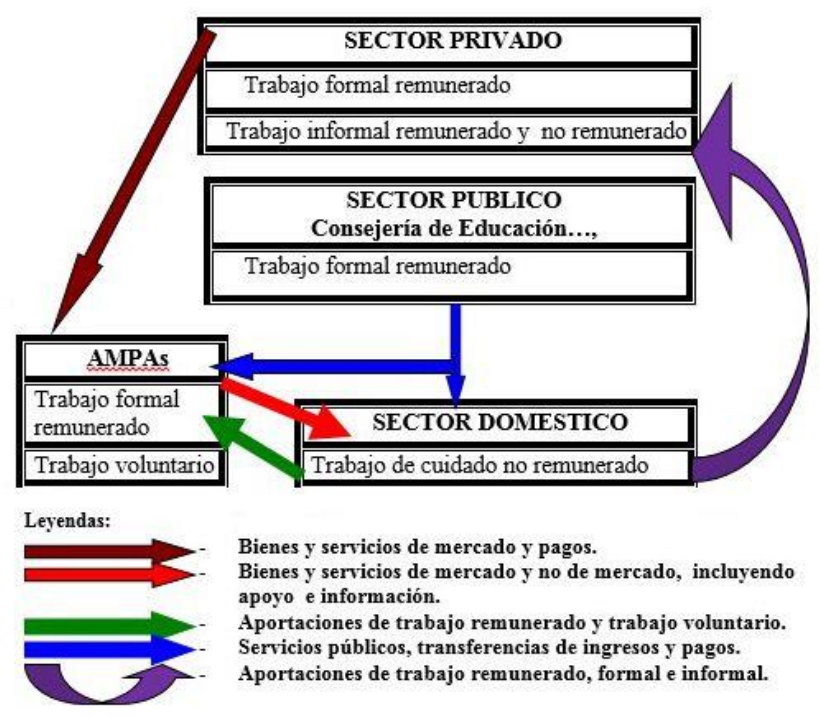

Fuente: Elaboración propia. Disgnóstico del Programa de Acogida Temprana. 
En el programa de Acogida temprana existe, por una parte, una inicial transferencia de servicios públicos (flecha azul) hacia el sector doméstico, o sea la propia ejecución del programa. Por otra parte, se identifican otras transferencias de bienes y servicios hacia el sector AMPAs (transferencia de ingresos, subvención-pagos) que tiene una doble vertiente. La primera es la del mercado (flecha rojo oscuro) y desde la cual las AMPAs se encargan de contratar empresas para que lleven a cabo las tareas de acogida y cuidado. Esto último implica aportaciones de trabajo remunerado desde el sector doméstico al privado (flecha púrpura). La segunda vertiente es la de la propia gestión de las AMPAs, de voluntariado, que implica aportaciones desde el sector doméstico (flecha verde) que es recompensada con otras desde las AMPAs (flecha roja).

Lo anterior desvela la gran contradicción de este programa: ciertamente, tiene un impacto positivo en los agentes directos, pero, tal y como está diseñado, incide negativamente en otros agentes, como las AMPAs o las condiciones de trabajo de las empresas adjudicatarias. Las AMPAs son espacios altamente feminizados, donde parece existir una importante segregación sexual respecto a la dedicación en horas de trabajo no remunerado; por tanto, el programa refuerza roles de cuidado y atención a la infancia, a través del voluntariado y usos del tiempo no mercantilizado. Con toda la información precedente, se diseñaron una serie de indicadores de género con el objetivo de ser puestos en marcha en el siguiente periodo. Resumimos en la siguiente tabla algunos de los más relevantes del programa de Acogida temprana.

\section{Tabla 1. Indicadores del Programa de Acogida Temprana}

\begin{tabular}{|l|l|}
\hline \multicolumn{1}{|c|}{ INDICADORES ESPECIFICOS } & \multicolumn{1}{c|}{ FUENTES } \\
\hline Desayunos escolares en el programa. & $\begin{array}{l}\text { Planificación y Estadística de la } \\
\text { Consejería. Requiere ampliar variables. }\end{array}$ \\
\hline $\begin{array}{l}\text { Familias monoparentales y monomarentales solicitantes y } \\
\text { aprobadas en el programa. }\end{array}$ & $\begin{array}{l}\text { Informes del centro y de la Inspección } \\
\text { Educativa. Requiere sistematización }\end{array}$ \\
\hline $\begin{array}{l}\text { Participación de los progenitores en el mercado de trabajo y y } \\
\text { situación de empleo. }\end{array}$ & $\begin{array}{l}\text { En la solicitud de las familias. Requiere } \\
\text { sistematización }\end{array}$ \\
\hline $\begin{array}{l}\text { Participación del profesorado en el programa (sexo, } \\
\text { dedicación laboral y categorías). }\end{array}$ & $\begin{array}{l}\text { Planificación y Estadística de la } \\
\text { Consejería. Requiere sistematización }\end{array}$ \\
\hline $\begin{array}{l}\text { Características de las empresas que participan. } \\
\text { Configuración de las plantillas de las empresas participantes } \\
\text { (sexo, dedicación laboral, categorías, salarios, etc.). }\end{array}$ & $\begin{array}{l}\text { Informes del centro y de la Inspección } \\
\text { Educativa. Requiere sistematización. } \\
\text { sistematización. }\end{array}$ \\
\hline $\begin{array}{l}\text { Medidas de igualdad de oportunidades en las empresas. } \\
\text { Empresas adjudicatarias. Requiere } \\
\text { sistematización. }\end{array}$ \\
\hline $\begin{array}{l}\text { Participación del AMPA en la gestión del programa } \\
\text { (progenitores, horas, tareas, etc.) }\end{array}$ & $\begin{array}{l}\text { Informes de las AMPAs. Requiere } \\
\text { investigación. }\end{array}$ \\
\hline $\begin{array}{l}\text { Participación de los/las responsables de la gestión del } \\
\text { programa del AMPA en el mercado de trabajo (sexo, } \\
\text { dedicación laboral, categorías, etc.). }\end{array}$ & $\begin{array}{l}\text { Informes de las AMPAs. Requiere } \\
\text { investigación. }\end{array}$ \\
\hline
\end{tabular}

Fuente: Elaboración propia. Diagnóstico del Programa. 
Dichos indicadores muestran las principales carencias metodológicas y técnicas para la evaluación del programa y que, en resumen, se centraba en la falta de un enfoque de transversalidad y de la sistematización de fuentes.

\subsection{Seguimiento del programa}

El programa de Acogida Temprana se inició en plena crisis (2008) y su evolución ha sido compleja por los continuos cambios de gestión y las polémicas, especialmente entre la institución y los sindicatos. El proyecto IGECAN, y por tanto la implantación de los indicadores de género, se paralizó al año siguiente por falta de presupuesto. La continuidad se ha llevado a cabo por parte de la investigadora principal y a partir del seguimiento de algunos programas, entre ellos el de Acogida temprana.

A partir del curso 2012-2013 el Gobierno de Canarias comunica a los Centros educativos la necesidad de medidas extraordinarias de reducción del gasto que afecta, de modo especial, a este programa. Entre otras cuestiones, plantea que la gestión pase a las AMPAs y/o Ayuntamientos, financiada con fondos propios y estableciendo la posibilidad de cuotas proporcionales a los ingresos de las unidades familiares (tramos de renta). Finalmente, se decidió que las familias aportasen una cantidad mensual, que suele oscilar entre veinte y treinta euros; todo ello a pesar del conflicto generado entre el organismo, los sindicatos y las AMPAs. Muchos centros han tenido dificultades para implantar estos tramos, por la realidad de numerosas familias en situación de vulnerabilidad. Esto explica su apoyo económico y la flexibilidad ante la norma.

"Nosotros tenemos un setenta por ciento de los padres en paro, pero a éstos el paro les obliga a asistir a unos cursos, entre otras cosas porque están recibiendo alguna ayuda. La mayoría de estos cursos comienzan a las ocho de la mañana, por lo tanto, no pueden traer a sus niños al colegio ¿Cómo le voy a pedir yo a un padre que me pague veinte euros por esto, si están cobrando cuatrocientos euros de ayuda? (Miembro del AMPA, CEIP de Tenerife, 2014)".

A partir del 2014, alrededor de una cuarta parte del total de centros continúan beneficiándose de la medida. A pesar de las continuas quejas y de reiterarse la evaluación negativa del programa, la gestión es asumida casi completamente por las AMPAs, con el aporte de las familias. Los requisitos para el desarrollo de la medida han cambiado de modo importante, debido a la escasez de recursos. Por tanto, el gráfico expuesto arriba se transforma de modo importante, acentuando la carga de trabajo voluntario que afecta de modo especial a mujeres-madres miembros del AMPA. Por el seguimiento que se viene realizando al programa, hay otros dos efectos negativos: uno, la mayor visibilidad del tipo de trabajo que se ha denominado de tipo mixto/hibrido o de trayectoria mixta. Predominan los casos de transferencia del sector doméstico y privado al público, a través del doblete de mujeres que trabajan formalmente en otra actividad del centro (comedores, actividades extraescolares, etc.) y hacen multitareas o utilizan los tiempos entre las mismas para realizar actividades de voluntariado. Dos, el aumento de contratos precarios (una/dos 
horas diarias, por ejemplo) en diversos servicios educativos (especialmente en Acogida temprana, Actividades extraescolares y Comedores).

\section{Debilidades, oportunidades y contradicciones}

A través de esta evaluación específica pueden identificarse debilidades, oportunidades y contradicciones que afectan a la práctica del mainstreaming. Aquí lo haremos a través de cinco aspectos transversales, prioritarios para este tipo de análisis: la identificación de asuntos clave, la sensibilización, las herramientas metodológicas, la coordinación-participación y la relación entre lo público y lo privado.

\subsection{Identificación de asuntos clave}

La metodología cualitativa tiene la ventaja de apoyarse en los encuentros cara-a-cara y, por tanto, de bajar al terreno de los impactos reales de determinadas políticas y programas. Por tanto, nos ofrece la oportunidad de identificar los asuntos más relevantes que pueden y deben trasladarse al diseño de las políticas. Ciertamente, en los proyectos con enfoque de género hay temáticas repetidas: los estereotipos, el cuidado infantil, el trabajo invisible, etc.; pero a partir de las mismas es posible identificar sub-temas o sub-sectores que hayan podido quedar olvidados o relegados. Por ejemplo, en el programa de Acogida temprana esto sucedía con los desayunos escolares: servicio complementario, directamente relacionado con el cuidado de la infancia y con el trabajo de los progenitores. En Canarias es de especial importancia por dos hechos: la elevada incidencia en las islas de enfermedades infantiles relacionadas con el equilibrio alimentario (Serra et al., 2003) y la crisis reciente, que afecta de modo especial a la alimentación de niños/as de familias de bajos ingresos económicos. Sin embargo, se carecía de datos sobre dicho servicio complementario, por lo cual se introdujeron indicadores de género sobre el mismo. De hecho, en el curso 2013-2014 el Gobierno de Canarias ha desarrollado medidas especiales en el servicio de comedores, para el alumnado de familias desfavorecidas o con bajos niveles de renta.

\subsection{La sensibilización}

El inicio de una línea de trabajo sobre impacto de género ya es representativo del nivel de sensibilización del organismo promotor, aunque básicamente a través de altos cargos que muestran la relevancia del feminismo institucional. Sin embargo, las resistencias también se hicieron visibles a lo largo del proyecto. Por parte del organismo se insistía en la continua formación de su personal en temas de género, tanto del empleado en la administración como del profesorado. Sin embargo, se carece de evaluaciones sobre dicha formación y, por tanto, se 
desconoce tanto el impacto real en la práctica de la Institución como el nivel de sensibilización de su personal. Paradójicamente otro tipo de agentes -con menos poder directo en la toma de decisiones- mostró una elevada sensibilidad con la temática del proyecto; es el caso de los/las participantes de las AMPAs, de directores/as de colegios e institutos, de los Consejos escolares, etc., que trabajan directamente con estos programas y que, por tanto, conocen cara-a-cara sus impactos reales.

Por tanto, la sensibilización es un elemento transversal que debe abordarse a partir de la cercanía con las temáticas clave. Se hace imprescindible que los organismos desarrollen estrategias para que el funcionariado, con tareas relevantes en determinadas políticas, salga de sus encierros burocráticos.

\subsection{Las herramientas metodológicas}

Para visibilizar todas estas carencias y apoyar la necesidad de cambios, la evaluación y el desarrollo de herramientas de trabajo resulta crucial, articulando los distintos niveles y comparando procesos. Por ejemplo, la realización de evaluaciones en varios programas y/o acciones permite la comparación de impactos, así como de sus trayectorias. Este ha sido el caso del proyecto realizado, dónde se puede contrastar los indicadores requeridos en cada uno de los programas, sus semejanzas y diferencias. Atender a la diacronía resulta de gran utilidad para identificar como les ha afectado hechos sincrónicos comunes: por ejemplo, las consecuencias de la crisis (recortes económicos) y los cambios de gestión. Este análisis procesual resulta esclarecedor para profundizar en los discursos y prácticas respecto a la transversalidad. En este caso, resulta ilustrativo que los recientes recortes hayan afectado de modo muy especial al único PIO: Acogida temprana.

Las técnicas utilizadas en el proyecto no resultan novedosas, aunque, en todo caso, si lo ha sido el enfoque multiestratégico en el uso de estadísticas, informes, trabajo de campo, análisis de presupuestos e indicadores de género, etc. Como se ha podido comprobar, la institución cuenta con fuentes para evaluar gran parte de los indicadores de género propuestos (informes de los centros y AMPAs, datos estadísticos, aunque no desagregados, etc.), aunque la mayoría no están sistematizadas. La explicación suele ser tan simple como que gran parte de estas herramientas se utilizan como meros instrumentos burocráticos, solicitándose para trámites y justificaciones varias, pero que luego ser archivan sin más. En otros casos, los datos pueden existir pero no interesa su difusión: este podría ser el caso de los datos de las empresas adjudicatarias de servicios. Por lo tanto, la transversalidad se muestra en el diseño de herramientas que atraviesen todos los niveles (presupuestos, indicadores con enfoque de género, etc.) y, por supuesto, en la transparencia y la difusión de los datos.

En todo caso indicar que, entre todas las técnicas utilizadas, el trabajo de campo ha mostrado su pertinencia para conocer y analizar percepciones, opiniones y soluciones que los diferentes agentes aportan. Por ejemplo, a través de la observación y las entrevistas se advierte el elevado 
conocimiento de algunos agentes (Consejo escolar, AMPAs, por ejemplo) de su realidad cotidiana, haciendo análisis de alto nivel reflexivo. De hecho, muchos Consejos escolares han aplicado una política de flexibilidad respecto a la aplicación de ciertas normas porque insistían en que éstas no se adaptaban a la realidad en sus ámbitos ${ }^{6}$. Algo similar ocurrió con las visitas a las empresas adjudicatarias, donde nuestra sola presencia y preguntas mostraba la irrupción inesperada en el construido equilibrio de intereses entre lo privado y lo público.

\subsection{La coordinación-participación}

La transversalidad requiere de una elevada reflexión sobre la coordinación y la participación, tanto a nivel teórico como práctico, claves para el desarrollo de estos procesos y la articulación entre los distintos niveles. Las estrategias para que agentes, ámbitos, modelos de actuación e intereses se interrelacionen tienen un elevado nivel de subjetividad e imaginación; sin embargo, el éxito y/o el fracaso de determinadas acciones y políticas suele explicarse a partir de cómo se diseñan, o no, protocolos de actuación y buenas prácticas. Un ejemplo es el mandato de la transversalidad. El mismo es algo ineludible en todo discurso político (leyes, planes, normativas), apoyándose en algunas de las herramientas más relevantes: comisiones interdepartamentales e interinstitucionales, formación para la igualdad, unidades de género, defensorías de igualdad, informes de impacto de género, observatorios de igualdad y consejos participativos de mujeres, etc.; sin embargo, las evaluaciones realizadas muestran una inserción más bien retórica, con normativas no vinculantes y la carencia de procesos participativos (Alonso, 2015).

En el caso de la Comunidad Autónoma Canaria, a partir del II Plan se menciona específicamente la estrategia de transversalidad o mainstreaming. Sin embargo, la misma no se articula ni en las distintas consejerías, ni en los niveles de actuación, siendo una amenaza para las futuras políticas de igualdad de género. Mencionaremos dos ejemplos. El primero de ellos se refiere a la creación desde el Gobierno de Canarias, entre el 2010 y 2013, de las denominadas Unidades de Modernización Administrativa (UMA). En el 2012 se constituyó dicha unidad en la Consejería de Presidencia, Justicia e Igualdad, donde está adscrito el Instituto Canario de Igualdad. Entre sus funciones se menciona: "El impulso, coordinación e implementación de la perspectiva de género en la planificación, gestión y evaluación en las políticas de la Consejería, en los términos que se establezcan en la normativa reguladora de las unidades de igualdad de género" (Consejería de Presidencia, Justicia e Igualdad, 2012). Curiosamente, la UMA en la Consejería de Educación, donde se inscribe el programa analizado, se creó algo antes, en el 2010, y entre sus funciones no se menciona la de implementar la perspectiva de género (Consejería de Educación, 2010). Por el contrario, en julio del 2014 se aprobó la Ley Canaria de Educación no

\footnotetext{
${ }^{6}$ La medida de Acogida temprana está dirigida únicamente a progenitores integrados en el mercado laboral formal y remunerado, aunque el Consejo escolar tiene cierta libertad de decisión. En la práctica, la mayor parte de los mismos son flexibles, beneficiando a familias en diferentes situaciones laborales y económicas, atendiendo a las situaciones de economía informal.
} 
Universitaria (Presidencia del Gobierno de Canarias, 2014) que sí menciona en su disposición adicional decimoctava un Plan para la igualdad de género en el sistema educativo, que deberá estar aprobado en el plazo de dos años. El otro ejemplo se centra en los presupuestos. No existe una estrategia coordinada y transversal respecto a los presupuestos con enfoque de género y desde la sociedad civil ha habido múltiples críticas sobre su falta de participación. La actual estrategia para el 2013-2020 tiene como uno de sus ejes estratégicos el de implantación de la transversalidad de género en la Administración de la Comunidad Autónoma de Canarias, dónde se plantean numerosos objetivos y actuaciones. Sin embargo, un análisis presupuestario del periodo 20132016 desvela que el $96 \%$ del presupuesto se asigna al eje de prevención y violencia de género, siendo el resto a coste cero.

Por tanto, la escasa participación de los diferentes agentes sociales en la toma de decisiones dibuja una práctica donde destacan los conflictos, sea por la escasa comunicación/coordinación, la indefinición de responsabilidades o los sentimientos de escasa valoración del papel de los diferentes agentes. En el caso del programa de Acogida temprana, las controversias entre el organismo, los sindicatos y los padres/madres ha sido una constante. Si activamos la escucha hacia los distintos agentes sociales implicados, se puede comprobar que algunas de sus mismas soluciones coinciden con la evaluación del programa: la excesiva burocratización, la carencia de coordinación e incentivos al profesorado, la necesidad de que el propio organismo asuma la responsabilidad de la gestión del programa, la mejora de las herramientas evaluativas y la sistematización, etc. En teoría existen herramientas de coordinación y participación; en la práctica se quedan en meros mandatos, ya que no se diseñan medidas específicas para su desarrollo (composición, estructura, frecuencia de reuniones, toma de decisiones, etc.).

En este sentido, el mismo proyecto inicial se planteó como algo externo a la institución, sin participación del personal del organismo, aunque con la máxima colaboración en el aporte de datos. Precisamente, la carencia de una estrategia de sensibilización, coordinación y participación fue la gran debilidad del proyecto inicial, ya que podría haber mejorado procesos de articulación transversal.

\subsection{Lo público y lo privado}

Concretamente nos referimos al proceso de privatización de servicios, que ha aumentado considerablemente en los últimos decenios. Resulta obvio que la gestión de lo público desde lo

público parte de referentes bien distintos de la gestión de lo público desde lo privado, lo asociativo o lo mixto-híbrido. La crisis actual -con importantes recortes en materia educativa y en programas de igualdad de oportunidades-, desdibuja el perfil y adscripción de trabajadores/as a diferentes sectores, como sucede con el sector ONGs/Asociativo y el sector privado y/o público. Todo esto afecta, de modo especial, a las mujeres.

En el ejemplo analizado se comprobó la carencia de datos sobre las empresas adjudicatarias, el perfil de los trabajadores/as, sus condiciones de trabajo, planes o incentivos a la igualdad, etc. 
Sin embargo, a través del trabajo de campo se pudo percibir diferencias entre las condiciones de trabajo entre áreas/programas. Por ejemplo: por una parte, todo el sector relacionado con el cuidado infantil, altamente feminizado (programas como Acogida temprana y de Comedores, por ejemplo); por otra parte, el sector del transporte (programa de Transporte escolar) altamente masculinizado y que ha tenido una relevante trayectoria en la mejora de sus convenios laborales. Por tanto, la privatización de los servicios públicos y otras estrategias que incluyen la participación privada (paternariados o patrocinios) deben contar con análisis transversales y comparativos. Las políticas no son neutrales y si queremos pasar del mandato de la transversalidad a la práctica de la misma debemos ser críticos: los binomios entre planes de igualdad y privatización descansan en la violencia del mercado de trabajo. Esto afecta de modo especial a las mujeres-madres-trabajadoras, materializándose en los tipos de contrato, la inestabilidad laboral y las condiciones de vida.

\section{Conclusiones}

\subsection{Las retóricas de la igualdad}

El proceso en la evaluación de programas y el enfoque cualitativo nos ubica en la arena del cara-a-cara con las políticas, visibilizando de modo potente las retóricas de la igualdad. La transversalidad ha sido un concepto criticado por su ambigüedad y polisemia, pero, como se puede comprobar, a través del análisis empírico puede describirse con mayor nitidez los múltiples significados del término. Por ello, hacer un recorrido desde las políticas a los programas -y viceversa- puede convertirse en una potente estrategia desde la que identificar debilidades, oportunidades y contradicciones en la práctica de la transversalidad. Este trayecto -de continua retroalimentación- puede realizarse a través de asuntos clave como la sensibilización, las herramientas metodológicas, la coordinación-participación, lo público versus privado, así como de identificar sub-temáticas relevantes en cada caso.

El programa que ha servido de ejemplo, Acogida temprana, refleja, desde su misma razón de ser, la lucha -estructural y cotidiana- entre conciliación y trabajo, entre lo privado y lo público. La incorporación de las mujeres al mundo laboral implica redefinir tiempos, roles y las relaciones familiares-sociales, en especial de las clases sociales que están condicionadas por la necesidad de un doble ingreso (Carrillo y López, 2011). Las políticas públicas no son neutrales y la transversalidad se visibiliza en la valentía que, desde los poderes públicos, se tenga para hacer frente a las estructuras sociales, económicas, culturales, de desigualdad. Estos son los dilemas que tenemos que afrontar desde el enfoque de género, el feminismo institucional o las mismas paradojas de la maternidad (Allen, 2005). 


\subsection{Aportes teórico-metodológicos y de intervención}

Las conclusiones del análisis realizado aportan reflexiones teóricas, metodológicas y de acción desde las políticas públicas. Queda patente que la opción elegida para desarrollar políticas de igualdad de oportunidades entre mujeres y hombres ha sido la de implementar proyectos y programas, o sea acciones específicas, sin el soporte de una estrategia de mainstreaming. Esto incide en las dificultades, y posiblemente resistencias (Bustelo y Lombardi, 2005) para trasladar la igualdad de género a todos los niveles, etapas y agentes del sistema. Un breve análisis de los mandatos, a nivel macro, y la evaluación de la praxis, a nivel micro, aporta una rica información sobre las retóricas y realidades en la supuesta aplicación del enfoque transversal. Ejemplos en el nivel macro, son las contradicciones descritas entre las UMAs y la reciente Ley Canaria de Educación, que visibilizan que la supuesta institucionalización del enfoque de la igualdad de género carece de diseminación. A nivel micro, se ha descrito el impacto y las carencias en la sensibilización, coordinación-participación, herramientas de trabajo y la atención a los agentes y sectores implicados. Por último, el seguimiento del programa ha desvelado las contradicciones entre los discursos sobre la transversalidad y las agendas públicas en tiempos de crisis. El cambio del programa de Acogida temprana, primero de tipo universalista a otro por tramos y, luego, a ser gestionado por organismos externos (AMPAs, Ayuntamientos, etc.) es un claro ejemplo. El proceso no ha hecho más que acentuar el trabajo invisible, la precariedad de las mujeres-madrestrabajadoras y, también, las contradicciones entre los mandatos de transversalidad-igualdad de oportunidades y las prácticas desveladas a través de la gestión del programa.

Todo lo anterior hace que volvamos a defender el marco teórico emprendido por el feminismo y la economía comprensiva, reelaborándolo a partir de las nuevas realidades, algo necesario para avanzar desde las retóricas a las prácticas transversales en materia de igualdad de oportunidades.

\section{BIBLIOGRAFÍA}

- Allen, Ann (2005): Feminism and Motherhood in Western Europe, 1890-1970: The Maternal Dilemma. Nueva York: Palgrave Macmillan.

- Aliaga, Christel (2006): "How is the Time of Women and Men Distributed in Europe?". En: Population and Social Conditions, vol. 2, pp. 1-12.

- Alonso, Alba (2015): El mainstreaming de género en España. Hacia un compromiso transversal con la igualdad. Valencia: Tirant lo Blanc.

- Ainscow, Mel; Dyson, Alan; Goldrick, Sue y West, Mel (2013): "Promoviendo la equidad en educación”. En: Revista de Investigación en Educación, vol. 11, nº 3, pp. 44-56. 
- Artis, Julie y Pavalko, Eliza (2003): "Explaining the Decline in Women's Household Labor: Individual Change and Cohort Differences”. En: Journal of Marriage and Family, vol. 65, pp. 746-761.

- Biencinto, Natalia y González, Ángeles (2010): La transversalidad de género: métodos y técnicas. Instituto Andaluz de la Mujer.

- Bognar, Greg (2005): “The Concept of Quality of Live”. En: Social Theory and Practice, vol. $31, n^{\circ} .4$, pp. 561-580.

- Booth, Cathryn et al (2002): “Child- Care Usage and Mother- Infant Quality time”. En: Journal of Marriage and Family, vol. 64, n. 1, pp. 16-26.

- Budlender, Debbie; Sharp, Ronda y Allen, Kerri (1998): How to do a gender sensitive budget analysis: Contemporary Research and Practice. London: Commonwealth Secretariat y AusAid.

- Budlender, Debbie y Hewitt, Guy (2003): Engendering Budgets: A Practitioners' Guide to Understanding and Implementing Gender-responsive Budgets. Commonwealth Secretariat.

- Bustelo, María y Lombardi, Emanuela (2005): "Mainstreaming de género y análisis de los diferentes 'marcos interpretativos' de las políticas de igualdad en Europa: el proyecto MAGEEQ”. En: Aequalitas. Revista Jurídica de Igualdad entre Mujeres y Hombres, $\mathrm{n}^{\circ}$. 17, pp. 15-26.

- Bustelo, Maria (2009): "Intersectionality faces the strong gender norm". En: International Feminist Journal of Politics, vol. 22, $\mathrm{n}^{\circ}$. 4, pp.530-546.

- Carrillo, Dolores y López, María José (2011): “Conciliación laboral y familiar y protección social en España”. En: Revue du REDIF, vol. 4, pp. 10-16.

- Cagatay, Ninufer; Keklik, Mümtaz; Lal, Rhadika y Lang, James (2000): Budgets as if People Mattered: Democratizing Macroeconomic Policies. New York: UNDP/SEPED.

- CEPAL (2006): Guía de asistencia técnica para la producción y el uso de los indicadores de género. Chile: Unidad Mujer y Desarrollo CEPAL.

- Consejería de Educación del Gobierno de Canarias (2008): "Resolución conjunta de la dirección general de promoción educativa y de la dirección general de personal de la consejería de educación, universidades, cultura y deportes, de fecha 18 de diciembre de 2008, por la que se aprueban instrucciones complementarias para la aplicación de las medidas de mejora de los rendimientos escolares y de la calidad del sistema educativo: acogida temprana y refuerzo educativo, curso escolar 2008/2009”. Disponible en: https://www.gobiernodecanarias.org/educacion/udg/pro/plan/docs/ResolucionINSTRUCCIONE SMedidasCALIDAD.pdf [28/04/2017].

- Consejería de Educación del Gobierno de Canarias (2010): “ORDEN de 31 de agosto de 2010, por la que se crea la Unidad de Modernización Administrativa (UMA), adscrita a la Secretaría General Técnica del Departamento". Disponible en: http://www.gobiernodecanarias.org/boc/2010/179/002.html [28/04/2017].

- Consejería de Presidencia, Justicia e Igualdad del Gobierno de Canarias (2012): “ORDEN de 4 de mayo de 2012, por la que se crea la Unidad de Modernización Administrativa de la 
Consejería de Presidencia, Justicia e Igualdad". Disponible en: file:///C:/Users/Carmen/Downloads/boc-a-2012-091-2392.pdf [28/04/2017].

- Consejo de Europa (1999): Mainstreaming de género. Marco conceptual, metodología y presentación de "buenas prácticas". Informe final de las actividades del grupo de especialistas en mainstreaming. Madrid: Instituto de la Mujer, Ministerio de Trabajo.

- Elson, Diane (2002): Iniciativas de presupuestos sensibles al género: dimensiones claves y ejemplos prácticos. Chile: Gobierno de Chile, PNUD, UNIFEM, GTZ.

- England, Paula (2005): "Emerging Theories of Care Work". En: Annual Review of Sociology, vol. 31, pp. 381-399.

- Ferber, Marianne y Nelson, Julie. (2004): Más allá del Hombre Económico. Madrid: Cátedra.

- Folbre, Nancy (2006): "Measuring Care: Gender, Empowerment, and the Care Economy".

En: Journal of Human Development, vol. 7, nº 2, pp. 183-199.

. (2009): “Time Use and Living Standards". En: Social Indicators Research, vol. 93, n. 1, pp. 77-83.

- Gálvez, Lina y Torres, Juan (2010): Desigualdades. Mujeres y hombres en la crisis financiera. Barcelona: Icaria.

- Gobierno de Canarias (n.d.): "Estrategia para la igualdad de mujeres y hombres 2013-2020". Gobierno de Canarias consejería de presidencia, justicia e igualdad. Disponible en: http://www.gobiernodecanarias.org/opencms8/export/sites/icigualdad/resources/documentacion/ Planes/Estrategia_Igualdad_2013-2020.pdf [28/04/2017].

- Gornick, Janet y Meyers, Marcia (2003): Families that Work. Policies for Reconciling Parenthood and Employment. New York: Russell Sage Fundation.

- Goñi-Legaz, Salone; Ollo-López, Andrea y Bayo-Moriones, Alberto (2010): "The Division of Household Labor in Spanish Dual Earner Couples: Testing Three Theories”. En: Sex Roles, vol. 63, pp. 515-529.

- Greenstein, Theodore (2000): "Economic Dependence, Gender and the Division of Labor in the Home: A Replication and Extension”. En: Journal of Marriage and Family, vol. 62, n. 2, pp. 322-335.

- Haddad, Lenira (2002): An Integrated Approach to Early Childhood Education and Care. UNESCO. Toronto: University of Toronto.

- Htun, Mala y Weldon, Laurel (2010): "When do Governments Promote Women's Rights? A Framework for the Comparative Analysis of Sex Equality Policy". En: Perspectives on Politics, vol. 8, no. 1, pp. 201-216.

- Hugher, Michael (2006): “Affect, Meaning and Quality of Life”. En: Social Forces, vol. 85, n'. 2, pp. 611-629.

- Instituto Canario de estadística (2011): Mujeres y Hombres en Canarias 2011. Canarias: ISTAC. 
- Krapf, Sandra (2014): "Who Uses Public Childcare for 2-Year-Old Children? Coherent Family Policies and Usage Patterns in Sweden, Finland and Western German". En: International Journal of Social Welfare, vol. 23, nº.1, pp. 25-40.

- Lombardo, Emanuela (2003): "El Mainstreaming. La aplicación de la transversalidad en la Unión Europea”. En: Aequalitas. Revista Jurídica de Igualdad de Oportunidades entre Mujeres y Hombres, $n^{\circ} 13$, pp. 6-11.

- Mclanahan, Sara y Percheski, Christine (2008): "Family Structure and the Reproduction of Inequalities”. En: Annual Review of Sociology, vol. 34, pp. 257-276.

- Noonan, Mary (2001): “The Impact of Domestic Work on Men’s and Women's Wages”. En: Journal of Marriage and Family, vol. 63, pp. 134-1145.

- Nussbaum, Martha (2012): Crear capacidades. Propuesta para el desarrollo humano. Barcelona: Espasa Libros.

- OECD (2005). Babies and Bosses: Reconciling Work and Family Life. Volume 4: Canada, Finland, Sweden and the United Kingdom. Paris: Organization for Economic Co-operation and Development (OECD).

- Rodríguez, María José (2013): "El pluralismo asociativo femenino en municipios españoles. Propuesta de tipología". En: Reis, nº 142, Abril-Junio, pp. 123-140. Disponible en: http://www.reis.cis.es/REIS/PDF/REIS_142_071365512543768.pdf [28/04/2017].

- Presidencia del Gobierno de Canarias (2014): "LEY 6/2014, de 25 de julio, Canaria de Educación no Universitaria". Disponible en: http://www.gobcan.es/boc/2014/152/002.html. [28/04/2017].

- Sayer, Liana (2005): “Gender, Time and Inequality: Trend in Women's and Men's Paid Work, Unpaid Work and Free Time”. En: Social Forces, vol. 84, n. 1, pp. 285-303.

- Sen, Amartya. (2007): Elección colectiva y bienestar social. Madrid: Alianza Editorial.

- Serra, Luis et al. (2003): "Obesidad infantil y juvenil en España. Resultados del estudio enKid (1998-2000)". En: Med Clin, vol. 121, no. 19, pp. 725-32.

- Stetson, Dorothy y Mazur, Amy (1995): Comparative State Feminism. Thousand Oaks, CA: Sage.

- Torns, Teresa; Borrás, Vicent; Moreno, Sara y Recio, Carolina. (2008): "Las políticas de tiempo en Europa”. En: Revista Papers, nº. 49, pp. 77-84.

- UNIFEM. (2000): El progreso de las mujeres en el mundo 2000. Nueva York: Fondo de Desarrollo de las Naciones Unidas para la mujer.

- Vandenbroeck, Michael; Geens, Naomi y Berten, Hans (2014): “The Impact of Policy Measures and Coaching on the Availability and Accessibility of Early Chid Care: A longitudinal Study”. En: International Journal of Social Welfare, vol. 23, pp. 69-79.

- Villota, Paloma de (dir.) (2008): Diseño de la estrategia que permita la integración de la perspectiva de género en los presupuestos públicos. Madrid: Instituto de la mujer. 